\title{
Aproximación preliminar al sintagma fonológico en español
}

\author{
Nuria Polo Cano \\ Universidad Nacional de Educación a Distancia (UNED) \\ nuriapolo@flog.uned.es
}

\begin{abstract}
Submitted: 28/07/2014. Accepted: 26/06/2015. Available on line: 31/03/2016
Citation / Cómo citar este artículo: Polo Cano, N. Aproximación preliminar al sintagma fonológico en español. Loquens, 2(2), e020. doi: http://dx.doi.org/10.3989/loquens.2015.020
\end{abstract}

RESUMEN: A pesar de que la jerarquía prosódica se propuso hace ya varios años, no es mucho el trabajo realizado hasta el momento sobre la caracterización de los constituyentes superiores de la jerarquía en español. Este trabajo propone utilizar dos procesos segmentales conocidos en esta lengua (la espirantización de las oclusivas sonoras y la sonorización de la fricativa alveolar sorda en posición de coda) como parámetros válidos en la delimitación de los sintagmas fonológicos, hasta ahora sin evidencia en esta lengua.

Para ello, se han utilizado datos de dos informantes de la variedad centropeninsular y se han grabado emisiones de /b/, /d/, /g/ y /s/ de las distintas estructuras sintácticas posibles en español, con un total de 334 estímulos de habla leída.

Solo se ha podido establecer una linde prosódica de sintagma fonológico entre sujeto y verbo, sobre todo con sujetos largos. Las fronteras prosódicas descubiertas en las otras estructuras sintácticas analizadas se pueden deber a delimitaciones prosódicas en niveles prosódicos inferiores o superiores (palabras prosódicas y sintagmas entonativos respectivamente).

Palabras clave: jerarquía prosódica; oclusivas sonoras; fricativa alveolar; español

ABSTRACT: A preliminary approach to phonological phrase in Spanish.- Although the prosodic hierarchy has been well established in phonological theory for decades, little empirical work on its higher constituents has been done on Spanish. This study examines two well-known phonological processes in Spanish (spirantization of voiced plosives and fricative sibilant voicing in coda position) as possible parameters in the delimitation of phonological phrases.

The data analyzed have been gathered from two native Spanish speakers from the Central Peninsular variety of Spanish, who read 334 stimuli created for that purpose.

The results suggest that there is a phonological phrase boundary between the subject and the verb, especially in long subjects. Other syntactic structures seem to be mapped as prosodic constituents of lower or higher levels (prosodic words and intonational phrases, respectively).

Keywords: prosodic hierarchy; voiced plosives; alveolar fricative; Spanish

\section{INTRODUCCIÓN}

El presente trabajo se formula como una primera aproximación al estudio del sintagma fonológico (phonological phrase) en español ${ }^{1}$. El objetivo principal del trabajo es utilizar dos de los procesos segmentales comunes en español (la espirantización de las oclusivas sonoras y la sonorización de la fricativa alveolar sorda en posición de coda) como pruebas empíricas objetivas para delimitar el sintagma fonológico, muy poco estudiado en esta lengua, ya que a pesar del trabajo realizado en fonología general sobre la jerarquía prosódica, el sintagma fonológico no es uno de los constituyentes que más ha centrado el interés en el marco de la fonología española.

${ }^{1}$ Si no se indica lo contrario, las traducciones son propias.

Copyright: (C) 2015 CSIC This is an open-access article distributed under the terms of the Creative Commons Attribution-Non Commercial (by-nc) Spain 3.0 License. 
El sintagma fonológico es un constituyente medio entre la palabra prosódica y el sintagma entonativo: por ejemplo, en he visto tres caballos muy oscuros hay tres sintagmas fonológicos: he visto, tres caballos, muy oscuros; y en no puedo decirte cuántos hombres estábamos aquí, también hay tres: no puedo decirte, cuántos hombres, estábamos aquí (ejemplos propuestos para el español a partir de los de Nespor y Vogel, 1986).

No obstante, parece que es un constituyente polémico, ya que no solo los autores cuestionan su existencia, sino que ni tan siquiera se ponen de acuerdo en su denominación. Por ejemplo, en estas páginas se ha traducido como sintagma fonológico el phonological phrase de Hayes, 1995; Nespor y Vogel, 1986/1994; y Selkirk, 1978/1981. Posteriormente Selkirk propuso el nombre de sintagma fonológico mayor (major phonological phrase; Selkirk, 1986, 2001), e incluso el de sintagma fonológico menor (minor phonological phrase; Selkirk, 1986; Selkirk y Tateishi, 1988, 1991). Beckman y Pierrehumbert (1986), por su parte, hablan de sintagma [entonativo] intermedio (intermediate phrase). A todos los efectos en estas páginas, dicho constituyente se denomina sintagma fonológico ${ }^{2}$.

Por un lado, este tema resulta de gran interés en el marco de los estudios sobre teoría fonológica, pues no existe ningún análisis moderno que aborde el sintagma fonológico en español que ofrezca pruebas segmentales de su existencia en esta lengua, aunque Gili Gaya (1950/1975), por ejemplo, ya intuyó esta división prosódica en su planteamiento. Los únicos trabajos que se conocen hasta el momento utilizan criterios sintácticos y suprasegmentales para definir los sintagmas fonológicos, como se explica más adelante. Por otro, aunque los procesos segmentales del español han recibido mucha atención, no es el caso de la interacción entre fronteras prosódicas y procesos fonológicos. Por ello, dado este vacío en la bibliografía, se propone el presente trabajo.

La hipótesis desde la que se ha partido se basa en que, si el sintagma fonológico existe en español, será susceptible de delimitación a través de marcas fonológicas segmentales, objetivamente analizables. Para llevarlo a cabo, se han elegido, de entre los distintos posibles, el proceso de espirantización que se produce en las obstruyentes oclusivas sonoras en determinados contextos, y la sonorización de la fricativa alveolar en posición de coda que ocurre ante consonantes sonoras, procesos ambos largamente documentados en español (Real Academia Española, 2011).

\subsection{La jerarquía prosódica}

Dentro de las teorías fonológicas modernas, donde la posición del segmento, y no el segmento en sí, es el epi- centro de las representaciones fonológicas, nace la fonología prosódica, que se desarrolla a partir de la postulación de una jerarquía prosódica universal. Dicha teoría define plantillas prosódicas en las que se inserta la información fonológica (segmental), morfológica y sintáctica en la representación prosódica de cualquier lengua ${ }^{3}$.

Nespor y Vogel (1986/1994) son unas de las primeras personas que han trabajado en este modelo concibiéndolo como una «teoría que organiza una secuencia de lengua determinada en una serie de constituyentes fonológicos jerárquicamente distribuidos en función de ciertas reglas postléxicas» (p. 18). Para cumplir estos dos requisitos, universalidad y jerarquía, dichas autoras establecen cuatro principios fundamentales que la cadena fónica o prosódica debe respetar (Nespor y Vogel, 1986/1994, p. 19) y que se pueden resumir de la siguiente manera:

- Principio 1. Cada unidad de la estructura jerárquica se compone de una o más unidades de la categoría inmediatamente inferior.

- Principio 2. Cada unidad perteneciente a un nivel de la jerarquía debe estar incluida en la unidad inmediatamente superior de la cual forma parte.

- Principio 3. Las estructuras jerárquicas de la fonología prosódica son de ramificación $n$-aria ${ }^{4}$.

- Principio 4. La relación de prominencia relativa entre los nudos hermanos se caracteriza por la asignación del valor «fuerte» a uno de los nudos y el valor «débil» al resto.

Estos cuatro principios ya estaban presentes de alguna forma en la hipótesis de estratificación rigurosa (strict layer hypothesis), propuesta por Selkirk dos años antes (1984).

Con el desarrollo de la morfología prosódica propuesta por McCarthy y Prince (1986), a esta descripción se añade un mecanismo explicativo nuevo mediante la noción de alineamiento (alignment), por la que la jerarquía prosódica debe sustentarse en una restricción general que obliga a alinear los elementos que la componen desde el margen izquierdo de la secuencia, avanzando hacia la derecha del enunciado. La alineación de los bordes de los ámbitos morfológicos y prosódicos resulta decisiva en varios procesos fonológicos (y morfofonológicos) como los que se analizan en las presentes páginas (Pater y Paradis, 1996, p. 543).

No obstante, una de las asunciones básicas en la fonología prosódica es que no existe isomorfismo entre las estructuras sintáctica y prosódica. Así, la jerarquía prosódica se define por sus propios constituyentes prosódicos.

Se establecen seis unidades básicas que la conforman. De mayor a menor, son el enunciado fonológico (utteran-

\footnotetext{
${ }^{2}$ Véase Ito y Mester (2013) para una discusión sobre la necesidad de reducir todas las variantes a una sola, el sintagma fonológico.

${ }^{3}$ No obstante, autores como Pak (2008), Samuels (2011) o Scheer (2011) niegan la existencia de la jerarquía y postulan que no es necesario crearla en el marco de la teoría fonológica. Quizás una de las mayores críticas que se le han hecho al modelo es la falta de recursividad de los constituyentes, aunque no dejan de presentarse propuestas teóricas que han abogado por una estructura prosódica recursiva (Ito y Mester, 2009, 2013; Ladd, 1986; Selkirk, 2011, entre otros).

${ }^{4}$ Estas autoras consideran que las ramificaciones son ilimitadas (n-arias), mientras que Selkirk (1984) considera que son binarias.
} 
ce; EF), el sintagma entonativo (intonational phrase; E), el sintagma fonológico (phonological phrase; $\varphi$ ), la palabra prosódica o fonológica (prosodic word; $\omega$ ), el pie (foot; $\Sigma$ ) y la sílaba (syllable; $\sigma)$. La presente investigación se centra en el sintagma fonológico; tanto las unidades inferiores como las unidades superiores de la jerarquía quedan fuera de los objetivos perseguidos en estas páginas. En la Tabla 1 se recogen los componentes de la jerarquía aplicados a un ejemplo del español.

Tabla 1. Ejemplo de componentes de la jerarquía prosódica en español.

\begin{tabular}{|l|l|}
\hline \multicolumn{1}{|c|}{ Elementos } & \multicolumn{1}{c|}{ Ejemplos en español } \\
\hline Enunciado fonológico & $\begin{array}{l}\text { Cuando Marí llegó al } \\
\text { aeropuerto, su marido ya } \\
\text { había facturado las maletas. }\end{array}$ \\
\hline Sintagma entonativo & $\begin{array}{l}\text { su marido ya había } \\
\text { facturado las maletas }\end{array}$ \\
\hline Sintagma fonológico & su marido \\
\hline $\begin{array}{l}\text { Palabra prosódica o } \\
\text { fonológica }\end{array}$ & marido \\
\hline Pie & -rido \\
\hline Sílaba & -ri- \\
\hline
\end{tabular}

En general, todos los autores están más o menos de acuerdo con esta clasificación. No obstante, Nespor y Vogel (1986/1994) o Hayes (1989) proponen un nivel intermedio entre el sintagma fonológico y la palabra prosódica: el grupo clítico, porque existen fenómenos fonológicos que no presentan como dominio de aplicación ni la palabra prosódica ni el sintagma fonológico. No todos los autores, sin embargo, consideran que el grupo clítico es una categoría diferenciada, porque este nivel no se define por procesos fonológicos específicos de su rango (Booij, 1988; Peperkamp, 1997; Selkirk, 1984). Otros fonólogos, por su parte, proponen un nivel más entre la palabra prosódica y el sintagma fonológico, como la palabra prosódica máxima (Ito y Mester, 2009) o el grupo de palabras prosódicas (Vigário, 2010), para describir la prosodificación de las palabras compuestas, niveles que tampoco todos los autores aceptan (Elordieta, 2014).

Sobre la descripción de la jerarquía prosódica del español en particular hay poco trabajo realizado hasta el momento, aunque sobre las categorías inferiores, como la sílaba o los pies métricos, se cuenta con bastantes estudios. Hualde (2003) y Nibert (2000) proponen que en esta lengua existen cuatro niveles únicamente: el sintagma entonativo, el sintagma intermedio (intermediate phrase; I), el sintagma fonológico y la palabra prosódica. Estos autores, aunque consideran cuestionable la existencia del sintagma fonológico en español, abogan por su existencia en esta lengua, a pesar de que hasta el momento no se haya demostrado mediante pruebas segmentales empíricas (Hualde, 2003; Nibert, 2000; v. también Prieto, 2006).

\subsection{El sintagma fonológico}

Nespor y Vogel (1986/1994, p. 194 y sigs.) definen el sintagma fonológico $(\varphi)$ de la siguiente manera:

- Ámbito de $\varphi$ : contiene un núcleo léxico y todos los grupos clíticos de su lado no recursivo, hasta llegar al grupo clítico que alberga otro núcleo situado fuera de la proyección máxima del sintagma.

- Construcción de $\varphi$ : posee ramificación n-aria y abarca todos los grupos clíticos incluidos en una secuencia delimitada por la definición del ámbito del sintagma fonológico.

- Prominencia relativa de $\varphi$ : en las lenguas cuyos árboles sintácticos se ramifican a la derecha, el nudo situado más a la derecha dentro del sintagma fonológico recibe la etiqueta de «fuerte»; en cambio, en las lenguas cuyos árboles sintácticos se ramifican a la izquierda, es el nudo de más a la izquierda dentro del sintagma fonológico el que recibe la etiqueta de fuerte. A todos los nudos hermanos del nudo fuerte se les asigna la etiqueta de «débil».

En este constituyente se hace uso, por tanto, de nociones sintácticas más generales, como las de sintagma y núcleo sintagmático, que lo convierten en una categoría de interfaz. En las lenguas que incrustan oraciones a la derecha, como el español, cada sintagma fonológico está compuesto por el núcleo sintagmático y todo lo que le precede dentro del mismo sintagma. En estas, el nudo de más a la derecha es el fuerte y los demás son débiles. Según Nespor y Vogel (1986/1994):

El sintagma fonológico resultante constituye, pues, otra prueba de la falta de isomorfismo entre las jerarquías sintáctica y prosódica. [...] Se ha demostrado que los complementos se agrupan en un sintagma fonológico junto con el núcleo si se encuentran a un lado del núcleo, [el izquierdo], y que forman sintagmas fonológicos independientes si se encuentran en el lado contrario [el derecho] (p. 213) .

Así sucede en los ejemplos siguientes, cuyo núcleo está a la derecha: en la casa, a la casa, no hay nada, pequeño niño, está guardada.

A pesar de las críticas al modelo, la jerarquía prosódica debe existir como parte de las representaciones fonológicas, ya que, de no ser así, no se podrían explicar en numerosas lenguas algunas características fonotácticas

\footnotetext{
${ }^{5}$ Dresher (1996) especifica que los sintagmas fonológicos siguen la misma dirección que los árboles sintácticos. Por ello, solo los sintagmas fonológicos coinciden con los constituyentes sintácticos; los constituyentes inferiores no coinciden con los constituyentes sintácticos.
} 
segmentales prosódicamente bien construidas, o el ritmo y la asignación de acentos, entre otras, que solo se adscriben a determinados niveles. Por otro lado, la sintaxis no puede predecir por sí sola la manera en la que se organizan los enunciados orales, para lo cual son necesarios los constituyentes prosódicos, porque «son las estructuras fonológicas de los constituyentes prosódicos y no los constituyentes sintácticos los que determinan el patrón prosódico de una oración» (Nespor y Vogel, 1986/1994, p. 297). Esto es, las unidades prosódicas son independientes de las fronteras sintácticas y - lo que es más importante - existen pruebas de que algunas reglas fonológicas y morfofonológicas operan en dominios que no coinciden con constituyentes sintácticos ${ }^{6}$.

Por ello, cada una de estas unidades fonológicas se describe mediante reglas meramente fonológicas, tanto segmentales como suprasegmentales, que se aplican únicamente dentro de cada dominio prosódico. Así, se ha trabajado bastante en descubrir las reglas fonológicas cuyo ámbito de aplicación está delimitado por los márgenes de los constituyentes prosódicos en las diferentes lenguas.

Ahora bien, el sintagma fonológico es uno de los constituyentes que más se ha estudiado en otras lenguas (Selkirk y Tateishi, 1988, 1991; Truckenbrodt, 1995, 1999); no así en español. Por ejemplo, en francés la conocida liaison se produce solo en el ámbito del sintagma fonológico, lo cual prueba la existencia de este constituyente al menos en esa lengua. Así pues, en las distintas lenguas el sintagma fonológico se ha propuesto con base en procesos entonativos (Beckman y Pierrehumbert, 1986) y con base en procesos segmentales (Nespor y Vogel, 1986).

En lo que respecta al caso concreto del español, a nuestro entender no existe ningún trabajo sobre este constituyente que se aborde partiendo de los procesos segmentales y apenas se sabe nada sobre sus ámbitos de aplicación en esta lengua. De hecho, hasta el momento no se ha descubierto ninguna regla ni proceso fonológico que se aplique solo en este dominio y que, por ello, corrobore su existencia en español.

Los únicos trabajos publicados sobre este constituyente adoptan enfoques entonativos, como el de Hualde (2003), quien propone que, en español, el sintagma intermedio parece existir para evitar la ambigüedad de algunas estructuras sintácticas. Por ejemplo, una misma estructura sintáctica, como SVO, puede corresponderse con distintas estructuras informativas. En el ejemplo tomado de este autor (p. 168), en la oración La vieja lanza la amena- $z a$, cuya estructura es SVO, se observa ambigüedad al darse dos lecturas posibles: [la vieja]SN [lanza la amenaza]SV y [la vieja lanza]SN [la amenaza]SV. Se hace necesario, por tanto, que exista un constituyente prosódico medio, inferior al sintagma entonativo, mediante el cual se resuelva la ambigüedad: el sintagma fonológico. Igualmente, Prieto (2006) caracteriza los sintagmas fonológicos en español por medio de la presencia de determinados finales ascendentes en el constituyente.

En esta misma línea, Benet Parente (2012, pp. 14-15) propone que, en español, se distingue perceptivamente un corte prosódico al final de un dominio que no posee frontera tonal (por tanto, tiene que ser inferior al sintagma intermedio), pero contiene más de una palabra prosódica y, en consecuencia, no puede ser otro que el sintagma fonológico.

Estos ejemplos apoyan en cierta medida la existencia del sintagma fonológico, probada solo desde el punto de vista entonativo. Para corroborar desde el ámbito segmental estas intuiciones, se han seleccionado algunos procesos fonológicos que en español afectan típicamente a los segmentos, como prueba objetiva, independiente del contenido entonativo, de la existencia del constituyente en cuestión. No obstante, todas las categorías prosódicas no condicionan necesariamente todos los procesos segmentales, pero, cuando un constituyente lo hace, dichos procesos proporcionan pruebas claras de su existencia; por ello, este estudio resulta relevante.

\subsection{Procesos fonológicos en español objeto de análisis}

En este trabajo se parte de unos procesos fonológicos, ya descritos en relación con el español (RAE, 2011), para definir el ámbito del sintagma fonológico, puesto que, si este constituyente efectivamente existiera en español, sería susceptible de delimitación a través de marcas fonológicas segmentales.

En concreto, se ha elegido el proceso de espirantización que sufren las oclusivas sonoras en ciertos contextos en todos los dialectos del español ${ }^{7}$. Así, /b/, /d/, /g/, en ejemplos como bote, dama y gato, solo se realizan de manera oclusiva tras pausa o consonante nasal homorgánica, además de tras /1/ en el caso de /d/ (['bo.te], ['da.ma], ['ga. to]); mientras que en los demás contextos se realizan

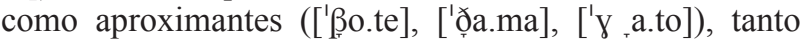
dentro como fuera de los límites de las palabras (Martínez Celdrán, 1984) $)^{8}$.

\footnotetext{
6 No obstante, esta propuesta se supera en Selkirk (1986), quien asume que los límites (izquierdo o derecho) de las proyecciones máximas se alinean con los límites (izquierdo o derecho) del sintagma fonológico. Según la lengua se decante por el parámetro de límite izquierdo o por el derecho, se proyectarán unos u otros sintagmas fonológicos.

7 Este fenómeno ha sido ampliamente abordado desde diferentes perspectivas: para un tratamiento desde la fonología autosegmental se puede consultar Goldsmith (1981/2000) o Harris (1984/2000), y se puede consultar González (2006) para un tratamiento desde el enfoque de la teoría de la optimidad. Recientemente se le ha dado cabida en el marco de la fonología articulatoria, por ejemplo, en Parrell (2011).

${ }^{8}$ Quilis ya demostró acústicamente que esta descripción no era tan categórica (1981, p. 224), pero no entraremos en estas páginas a debatir esta cuestión, pues escapa a los objetivos perseguidos. Más recientemente, Hualde, Shosted y Scarpace (2011) y Hualde, Simonet y Nadeu (2011) hablan de un continuum alofónico más que de una distribución complementaria. Para una revisión de esta discusión se puede consultar Parrell (2011).
} 
Por lo que respecta a la fricativa alveolar/s/, en español es sorda en posición de coda, pero puede sonorizarse ante consonantes sonoras (Quilis, 1993). Por ejemplo, en de/s/de es muy común la realización del segmento fricativo de forma sonora o sonorizada: de[z]de o de[s]de ${ }^{9}$. A pesar de que existe consenso general acerca de la existencia del fenómeno y de sus características, son más los datos basados en impresiones que los trabajos sistemáticos que profundicen en explicar esta cuestión (Campos-Astorkiza, 2010) ${ }^{10}$. Este proceso, al igual que el anterior, puede tener lugar dentro de una palabra o más allá de sus límites, como en los gatos [los. 'Y $\gamma_{\tau}$ a.tos] (para una revisión moderna, cf. Campos-Astorkiza, 2014).

Así, en el caso de las obstruyentes sonoras, si existe un límite prosódico a su izquierda, es probable que se produzca una realización oclusiva de dichos segmentos; por otra parte, en el caso de la fricativa alveolar/s/ en posición de coda, si existe un límite prosódico a su derecha, es probable que no se produzca la sonorización del segmento. Es decir, las palabras que contienen las oclusivas sonoras y la fricativa alveolar sorda se encuentran en un constituyente prosódico independiente del que contiene la palabra precedente (en el caso de las oclusivas sonoras) o siguiente (en el caso de la fricativa alveolar sorda). Se asume así que este constituyente prosódico es el sintagma fonológico.

Por ello, se ha decidido utilizar ambos procesos como pruebas válidas para intentar establecer la existencia del sintagma fonológico en español.

\section{METODOLOGÍA}

\subsection{Estímulos y sujetos}

Se construyó ad hoc una serie de estímulos, existentes en español, que contienen los cuatro segmentos en cuestión. Como la teoría prosódica admite la posibilidad de que diferentes estructuras sintácticas puedan dar lugar a un mismo patrón prosódico - ya que son las estructuras fonológicas de los constituyentes prosódicos las que determinan el patrón prosódico de una oración, no los constituyentes sintácticos (Nespor y Vogel, 1986/1994, p. 297)_, se seleccionaron contextos sintácticos básicos en español. Por lo tanto, las fronteras prosódicas de sintagma fonológico se corresponden con diferentes grados de cohesión sintáctica.

Así, se crearon estímulos en los que los contextos sintácticos que favorecen la realización de $/ \mathrm{b} /, / \mathrm{d} /, / \mathrm{g} /$ como oclusivas o aproximantes y la realización de /s/ como sorda o sonorizada se correspondieran con el grado de cohesión sintáctica entre la palabra que contiene estos segmentos y la palabra precedente (en el caso de /b, d, g/) o siguiente (en el caso de /s/). El grado de cohesión sintáctica entre dos palabras viene establecido por relaciones estructurales de complemento o adjunción, o por la pertenencia a las categorías léxica o funcional. El mayor grado de cohesión está representado por las secuencias determinante + nombre o clítico + verbo. Un grado de cohesión intermedio está representado por las secuencias nombre + adjetivo, verbo + objeto (tanto objetos cortos como largos) y sintagma nominal + sintagma preposicional ( $\mathrm{SN}+\mathrm{SPrep}$, como en el nieto de Manolo, tipo de ejemplo que no aparece en la Tabla 2), en el sentido de que la palabra de la izquierda es el núcleo y, la de la derecha inicial, el complemento de esta. El menor grado de cohesión sería el de la secuencia sujeto + verbo (tanto sujetos cortos como largos) o cláusula subordinada + cláusula principal, o cláusula principal + parentética.

De cada secuencia se propusieron tres ejemplos para cada una de las oclusivas /b/, /d/ y /g/, y tres para la fricativa alveolar /s/. En el caso de las fricativas, además, se añadió el contexto de nombre + adjetivo en plural (perros verdes) para controlar la posible diferencia entre una $-s$ léxica (perteneciente al sustantivo) y una $-s$ morfológica. Se controlaron los acentos en los estímulos para conseguir un equilibrio entre sílabas átonas y tónicas, ya que los estímulos se corresponden con palabras reales en las que no solo se buscó que la consonante en cuestión estuviera en el límite de los constituyentes, sino que en dichos límites hubiera igual número de sílabas átonas y tónicas, por lo que, por dar prioridad a los acentos y por usar palabras reales, no se pudieron controlar las vocales. Los estímulos se construyeron con $/ \mathrm{b} /, / \mathrm{d} / \mathrm{y} / \mathrm{g} /$ en el límite derecho de la frontera, y con /s/ en el límite izquierdo de la frontera. En el ámbito de la palabra, en ambos casos el límite aparece en su interior como margen de sílaba ( $b a b a$, desde). En el resto de los constituyentes sintácticos la frontera puede deberse a la existencia de una palabra, de un sintagma fonológico o de un constituyente superior. En la Tabla 2 se recogen, a modo de muestra, los ejemplos con la oclusiva bilabial.

Así pues, los estímulos creados fueron 84 para /b/ y el mismo número para $/ \mathrm{g} /, 88$ para $/ \mathrm{d} /$ (por la inclusión de la preposición de), y 78 para /s/, lo que supone un total de 334 estímulos, que fueron leídos por los participantes.

Los casos de $\mathrm{SN}+$ Sprep que se han analizado corresponden a preposiciones que empiezan por oclusiva dental sonora $/ \mathrm{d} /$, pues no existen preposiciones en español que empiecen por $/ \mathrm{b} / \mathrm{o} / \mathrm{g} /$ que formen la construcción $\mathrm{SN}+$ Sprep. No obstante, para poder llevar a cabo los análisis estadísticos y que toda la muestra fuera homogénea, se eliminaron de los recuentos finales.

Dado que se trata de un estudio preliminar, en el presente análisis solo se recogen los resultados de dos infor-

\footnotetext{
9 Se ha optado por la transcripción /s/ y añadir el diacrítico correspondiente de marca de sonoridad o la etiqueta de sonora para evitar confusiones, pues entendemos que /z/ se refiere al fonema y en español no tiene valor fonemático.

${ }_{10}$ Al igual que en el caso anterior, los trabajos recientes no observan una distinción categórica (Schmidt y Willis, 2011), pero de nuevo esta discusión se aleja de los objetivos perseguidos en estas páginas.
} 
Tabla 2. Ejemplos de los estímulos utilizados para /b/.

\begin{tabular}{|l|l|}
\hline \multicolumn{1}{|c|}{ Estructura sintáctica } & \multicolumn{1}{c|}{ Ejemplos } \\
\hline Palabra & baba \\
\hline Determinante + nombre & la boda \\
\hline Clítico + verbo & lo barre \\
\hline Nombre + adjetivo & gato bonito \\
\hline Verbo + objeto corto & decora bodas \\
\hline Verbo + objeto largo & fabrica botes de cristal reciclado \\
\hline Sujeto corto + verbo & Manolo baja las escaleras. \\
\hline Sujeto largo + verbo & $\begin{array}{l}\text { La hermana pequeña de Marina } \\
\text { barre el piso. }\end{array}$ \\
\hline Cláusula adverbial & $\begin{array}{l}\text { Cuando veniamos para la } \\
\text { facultad, vimos un accidente entre } \\
\text { un coche y una moto. }\end{array}$ \\
\hline Cláusula adjetiva & $\begin{array}{l}\text { María, a la que había tocado la } \\
\text { lotería en Navidad, vendió su casa } \\
\text { y dejó a su marido. }\end{array}$ \\
\hline
\end{tabular}

mantes, un hombre y una mujer, ambos hablantes de la variedad centropeninsular del español, y ambos con estudios superiores.

\subsection{Recogida de muestras}

Se pidió a los informantes que leyeran de la forma más natural posible los estímulos construidos. Fueron grabados en mono en un entorno silencioso mediante el programa Audacity (versión 2.0.5), con una frecuencia de muestreo de $44100 \mathrm{~Hz}$ y una resolución de 16 bits. Para ello se utilizó un micrófono cardioide Audio-technica MB $4 \mathrm{k}$ de condensador a unos $8-10 \mathrm{~cm}$ de la boca del informante. La señal se grabó directamente en un ordenador de mesa DELL Optiplex 360 en formato wav. Los estímulos se presentaron de manera aleatoria y se les pidió a los informantes que repitieran la lectura tres veces. Entre las repeticiones se dejaba transcurrir un tiempo para no fatigar a los participantes.

En total, se obtuvieron 768 muestras de obstruyentes oclusivas sonoras y 234 de fricativas alveolares de cada uno de los dos informantes, que suponen 1536 casos para las oclusivas y 468 casos para las fricativas, lo que suma un total de 2004 segmentos en producción leída, posteriormente analizados.

\subsection{Análisis de la muestras}

Para el tratamiento de los datos se usó el programa Praat (versión 5.3.82), y los segmentos en cuestión se anotaron mediante la opción TextGrid.

En el caso de las obstruyentes, se consideró que su realización era oclusiva si presentaban barra de explo- sión, y se etiquetaron como aproximantes o espirantizadas si no presentaban barra de explosión y además se apreciaban claramente los formantes. En el caso de la fricativa alveolar en posición de coda, se consideró sonora o sonorizada si presentaba barra de sonoridad.

Para los casos dudosos entre oclusiva o aproximante, los autores consultados coinciden en señalar que la oclusiva sonora dura más que la aproximante sonora, estableciendo distintos límites de duración e intensidad para delimitarlas: Parrell (2011), menos de $80 \mathrm{~ms}$, y Martínez Celdrán, menos de $55 \mathrm{~ms}$ para las aproximantes (Martínez Celdrán, 2013; Martínez Celdrán y Fernández Planas, 2007); y menor intensidad en el caso de la oclusiva que en el de la aproximante, con un descenso de energía, en el punto más bajo, por debajo de $60 \mathrm{~dB}$ en las oclusivas (Hualde, Shosted y Scarpace, 2011; Hualde, Simonet y Nadeu, 2011; Ortega-Llebaria, 2004).

Por tanto, siguiendo a estos autores, como criterio para etiquetar el segmento como oclusivo o como aproximante cuando su adscripción a una categoría u otra era dudosa, se consideró que para ser etiquetado como aproximante no debía presentar barra de explosión, debía durar menos de $55 \mathrm{~ms}$ y la energía no debía descender por debajo de $60 \mathrm{~dB}$. Las imágenes de las Figuras 1 y 2 muestran un ejemplo del criterio seguido: la Figura 1 muestra un ejemplo de realización oclusiva y, la Figura 2, de aproximante.

Por otra parte, en el caso de las fricativas, se consideró que existía sonorización cuando se apreciaban pulsos glotales regulares en la barra de sonoridad, tal como proponen Schmidt y Willis (2011) y Campos-Astorkiza

Figura 1. Oclusiva (papá barre)

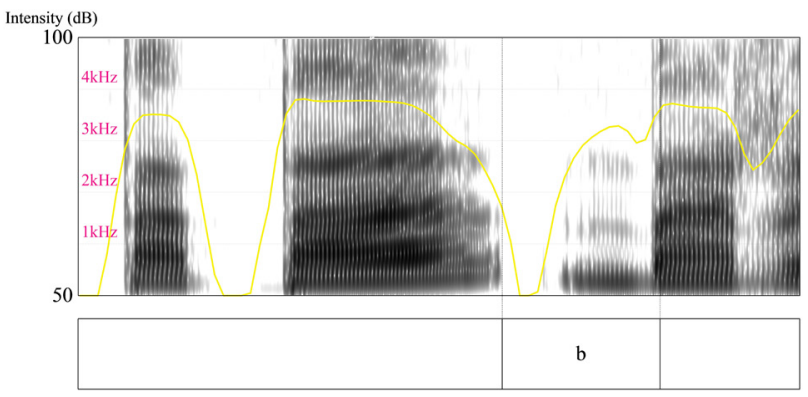

Figura 2. Aproximante (bebé).

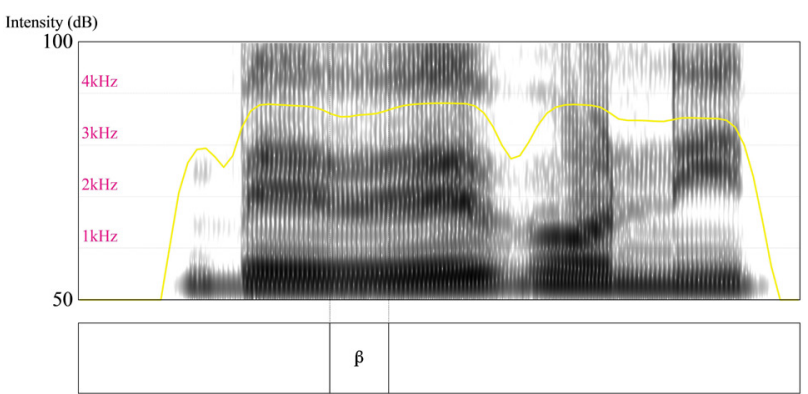


$(2014)^{11}$. En este sentido, se tuvo en cuenta la sonorización aunque fuera parcial y no afectara de manera completa al segmento ${ }^{12}$. En las Figuras 3 y 4 se presentan, respectivamente, un ejemplo de fricativa sorda y otro de fricativa sonora o sonorizada.

Cuando, en un contexto sintáctico determinado, se observa que al menos un $30 \%$ del total de las obstruyentes sonoras presenta una realización oclusiva, o que al menos un $30 \%$ del total de las fricativas alveolares en posición de coda se realizan como sordas, ello se ha interpretado como un índice de la existencia de una frontera prosódica que está bloqueando parcialmente el proceso de espirantización o de sonorización en cada caso ${ }^{13}$.

Tras obtener y preparar los datos, se llevó a cabo un análisis estadístico usando el programa SPSS (versión

Figura 3. Fricativa alveolar sorda (gustar).
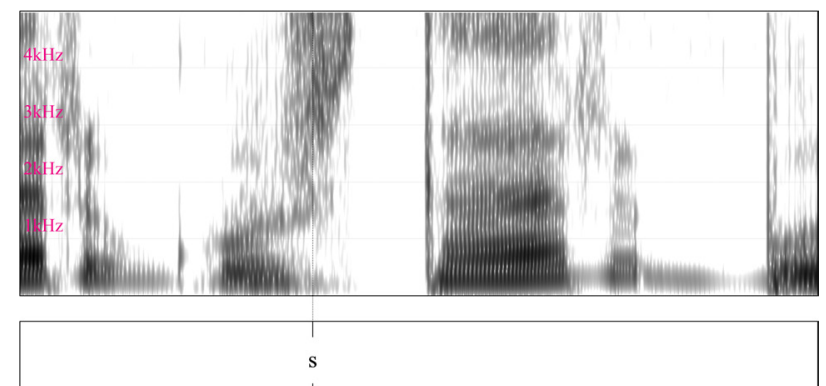

Figura 4. Fricativa alveolar sonora (los botes).

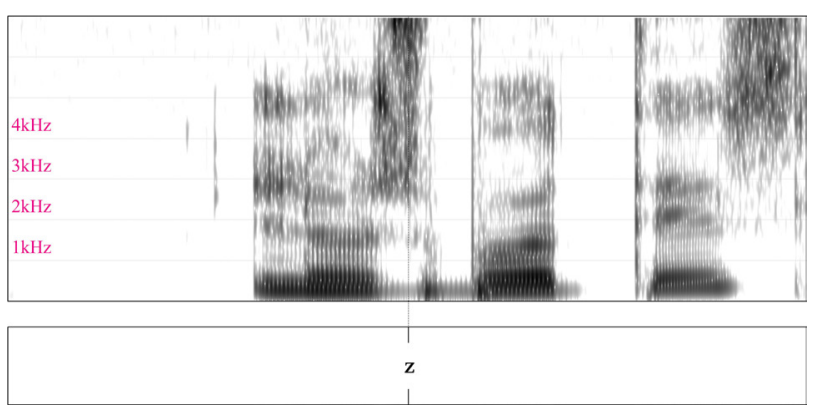

22.0.0). Para todos los casos se seleccionó la prueba de chi-cuadrado, pues las variables son siempre categóricas, siendo la variable dependiente la condición de oclusiva o de aproximante en las obstruyentes y de sorda o de sonora en las fricativas, y como factores la condición sintáctica, además de la comparación interhablante.

\section{ANÁLISIS Y RESULTADOS}

Para comprobar la coherencia de los resultados entre las tres repeticiones para el mismo sujeto, se llevó a cabo un análisis de fiabilidad usando el estadístico kappa de Fleiss. El estadístico kappa de marginalidad fija devuelve un índice de 0,51 para /b/, 0,63 para /d/, 0,65 para /g/ y 0,53 para /s/ en el caso de la mujer; en el caso del hombre, los índices son 0,64 para /b/, 0,63 para /d/, 0,73 para /g/ y 0,55 para /s/. Siguiendo a Landis y Koch (1977), esto supone una coherencia moderada en el caso de /b/y/s/ de la mujer; para el resto de casos, tanto de la mujer como del hombre, la coherencia es sustancial (kappa $>0,6$ ). Este resultado era esperable, pues las repeticiones fueron realizadas por la misma persona y existe variación intralocutor, aunque, según los resultados del test de fiabilidad, no es significativa y no responde más que a la propia variación del habla ${ }^{14}$.

No se observan diferencias significativas entre los dos sujetos, como muestra el análisis de chi-cuadrado realizado: $\chi^{2}(1)=2,0300 ; p=, 1542$ para las obstruyentes sonoras, y $\chi^{2}(1)=2,9926, p=, 0836$ para la fricativa alveolar. A pesar de ello y dado que la muestra de población es muy pequeña, los resultados se muestran por separado para los dos informantes (v. Tabla 3).

Tampoco se han encontrado diferencias significativas, por lo que se refiere a la presencia o a la ausencia de espirantización, al comparar los tres segmentos obstruyentes sonoros, tal como se desprende del análisis de chi-cuadrado realizado: $\chi^{2}(1)=0,0650, p=, 7988$ para la mujer, $\mathrm{y}$ $\chi^{2}(2)=3,7227, p=, 1555$ para el hombre. Esto supone que $/ \mathrm{b} /, / \mathrm{d} / \mathrm{y} / \mathrm{g} / \mathrm{se}$ comportan de manera similar, como una clase homogénea, al menos a efectos de los objetivos perseguidos en este trabajo. Por eso, los análisis se muestran asumiendo que /b/, /d/ y /g/ son un único grupo ${ }^{15}$.

\footnotetext{
11 Schmidt y Willis (2011) proponen una sonorización de al menos el 60 \% para que el segmento sea considerado sonoro. En nuestro caso se ha considerado sonoro aunque presente un porcentaje de sonorización inferior; ello implica que las conclusiones de este trabajo posiblemente no son tan restrictivas, al menos en lo que a este segmento se refiere.

12 Campos-Astorkiza (2015), entre otros, demuestra que la sonorización no es categórica, sino que normalmente suele ser parcial y gradual. De nuevo, no es el objetivo de estas páginas describir este proceso en sí mismo. Por ello, no se ha creído necesario realizar una normalización de los datos entre hablantes y comparar el porcentaje de sonorización en relación con la duración de la fricativa. Sin duda, ampliar este estudio conllevará revisar estas decisiones y aplicar estos u otros criterios.

13 Esta cifra es totalmente arbitraria: se podría haber tomado como referencia otro porcentaje, pero consideramos que un $30 \%$ sistemático de producción de oclusivas y de no sonorización de la fricativa alveolar es una cantidad considerable, que, al menos, hay que tener en cuenta.

${ }_{14}$ De todas formas, no existen datos suficientes sobre la variación intralocutor en español (Battaner et al., 2007).

15 Solamente en los casos de determinante + nombre, cuando el determinante es masculino (el dato), se han observado diferencias. No obstante, es de sobra conocido que en español la dental /d/ tras /1/ se realiza de forma oclusiva (Hualde, Shosted y Scarpace, 2011). Por ello, estos contextos también se han eliminado y solo se han considerado los casos en femenino, si bien llama la atención que en aquellos casos en que la labial /b/ entra en contacto con el determinante masculino se ha observado más producción de oclusivas de lo esperable (44 \% en la mujer y $22 \%$ en el hombre). No se han podido establecer conclusiones, pues en la bibliografía sobre el tema no se alude a la oclusión tras /1/ de forma similar a la de /d/ tras /1/ en español, de manera que el hecho puede deberse únicamente a que la lectura fuera más pausada en esos casos.
} 
En los casos de $\mathrm{SN}+$ Sprep, que, como ya se ha explicado (v. § 2.1), no se tuvieron en cuenta para los recuentos finales, el informante masculino realiza siempre una aproximante en este contexto para /d/ y la mujer realiza una aproximante en 23 de los 27 ítems $(85 \%)$ para los casos de $\mathrm{SN}+\mathrm{SPrep}$. La realización aproximante de la obstruyente /d/ indica que no existe una frontera prosódica entre el sintagma nominal precedente y el sintagma preposicional, pues el proceso no se bloquea; por ejemplo, en el hermano de Manolo, el hermano y de Manolo forman un único sintagma fonológico, al igual que ocurre en trabaja [no frontera] desde París.

El análisis de las distintas estructuras sintácticas que se ha llevado a cabo se recoge en la Tabla 3. Los resultados se presentan para cada informante por separado; además, por un lado se han agrupado los resultados de las tres obstruyentes sonoras, de las que se indican los casos de realización aproximante y, por el otro, se presentan los casos de realización sonora de la fricativa alveolar. Las celdas sombreadas indican que la espirantización o la sonorización se realiza en menos del $70 \%$ de los casos: por tanto, aparecen sombreados los contextos en los que ya aparecen oclusivas y no se sonoriza la fricativa alveolar en un mínimo del $30 \%$ del total de realizaciones, porcentaje que se ha tomado como umbral para establecer la existencia de una posible frontera prosódica que bloquee el proceso, en cada contexto sintáctico determinado en el presente análisis. La variación en el número de los grupos se debe a que se han controlado los acentos junto con los límites prosódicos (sílaba tónica o átona que colindan con una frontera prosódica a la izquierda y a la derecha).

Las Figuras 5 y 6 dan una idea más clara de cómo se distribuyen las producciones aproximantes y sonoras. En ellas se puede observar claramente una progresión descendente conforme a la cual el número de realizaciones aproximantes se va reduciendo a medida que disminuye el grado de cohesión sintáctica entre la palabra que contiene $/ \mathrm{b} \mathrm{d} \mathrm{g/} \mathrm{y} \mathrm{la} \mathrm{palabra} \mathrm{que} \mathrm{la} \mathrm{precede,} \mathrm{o} \mathrm{entre} \mathrm{la} \mathrm{palabra}$ que contiene /s/ final y la que la sigue.

Estos resultados muestran que, en las secuencias sintácticas de interior de palabra aislada, determinante + nombre, clítico + verbo, prácticamente el $100 \%$ las
Tabla 3. Realizaciones como aproximantes de las oclusivas y realizaciones sonoras de /s/ en función del contexto sintáctico. Las casillas sombreadas indican porcentajes de realización aproximante o sonora inferiores al $70 \%$.

\begin{tabular}{|l|c|c|c|c|}
\hline & \multicolumn{2}{|c|}{$\begin{array}{c}\text { Realización } \\
\text { aproximante de } \\
\text { /b, d, g/ }\end{array}$} & \multicolumn{2}{c|}{$\begin{array}{c}\text { Realización sonora } \\
\text { de /s/ }\end{array}$} \\
\hline $\begin{array}{c}\text { Estructura } \\
\text { sintáctica }\end{array}$ & Mujer & Hombre & Mujer & Hombre \\
\hline Palabras & $\begin{array}{c}53 / 54 \\
(98,1 \%)\end{array}$ & $\begin{array}{c}54 / 54 \\
(100 \%)\end{array}$ & $\begin{array}{c}17 / 18 \\
(94,4 \%)\end{array}$ & $\begin{array}{c}14 / 18 \\
(77,8 \%)\end{array}$ \\
\hline Det + nom. & $\begin{array}{c}48 / 54 \\
(88,9 \%)\end{array}$ & $\begin{array}{c}51 / 54 \\
(94,4 \%)\end{array}$ & $\begin{array}{c}18 / 18 \\
(100 \%)\end{array}$ & $\begin{array}{c}18 / 18 \\
(100 \%)\end{array}$ \\
\hline Clítico + V & $\begin{array}{c}54 / 54 \\
(100 \%)\end{array}$ & $\begin{array}{c}54 / 54 \\
(100 \%)\end{array}$ & $\begin{array}{c}18 / 18 \\
(100 \%)\end{array}$ & $\begin{array}{c}18 / 18 \\
(100 \%)\end{array}$ \\
\hline Nom. +adj. & $\begin{array}{c}106 / 108 \\
(98,1 \%)\end{array}$ & $\begin{array}{c}106 / 108 \\
(98,1 \%)\end{array}$ & $\begin{array}{c}23 / 36 \\
(63,9 \%)\end{array}$ & $\begin{array}{c}33 / 36 \\
(91,7 \%)\end{array}$ \\
\hline $\begin{array}{l}\text { Nom. + adj. } \\
\text { pl. }\end{array}$ & - & - & $\begin{array}{c}18 / 18 \\
(100 \%)\end{array}$ & $\begin{array}{c}15 / 18 \\
(83,3 \%)\end{array}$ \\
\hline V+ O corto & $\begin{array}{c}100 / 108 \\
(92,6 \%)\end{array}$ & $\begin{array}{c}104 / 108 \\
(96,3 \%)\end{array}$ & $\begin{array}{c}35 / 36 \\
(97,2 \%)\end{array}$ & $\begin{array}{c}35 / 36 \\
(97,2 \%)\end{array}$ \\
\hline V+ O largo & $\begin{array}{c}101 / 108 \\
(93,5 \%)\end{array}$ & $\begin{array}{c}100 / 108 \\
(92,6 \%)\end{array}$ & - & - \\
\hline Suj. corto + V & $\begin{array}{c}71 / 108 \\
(65,7 \%)\end{array}$ & $\begin{array}{c}97 / 108 \\
(89,8 \%)\end{array}$ & $\begin{array}{c}30 / 36 \\
(83,3 \%)\end{array}$ & $\begin{array}{c}34 / 36 \\
(94,4 \%)\end{array}$ \\
\hline $\begin{array}{l}\text { Suj. } \\
\text { largo + V }\end{array}$ & $\begin{array}{c}49 / 108 \\
(45,4 \%)\end{array}$ & $\begin{array}{c}59 / 108 \\
(54,6 \%)\end{array}$ & $\begin{array}{c}10 / 36 \\
(27,8 \%)\end{array}$ & $\begin{array}{c}26 / 36 \\
(72,2 \%)\end{array}$ \\
\hline $\begin{array}{l}\text { Cláusulas } \\
\text { adverbiales }\end{array}$ & $\begin{array}{c}0 / 27 \\
(0 \%)\end{array}$ & $\begin{array}{c}0 / 27 \\
(0 \%)\end{array}$ & $\begin{array}{c}0 / 27 \\
(0 \%)\end{array}$ & $\begin{array}{c}0 / 27 \\
(0 \%)\end{array}$ \\
\hline $\begin{array}{l}\text { Cláusulas } \\
\text { adjetivas }\end{array}$ & $\begin{array}{c}0 / 27 \\
(0 \%)\end{array}$ & $\begin{array}{c}0 / 27 \\
(0 \%)\end{array}$ & $\begin{array}{c}0 / 27 \\
(0 \%)\end{array}$ & $\begin{array}{c}0 / 27 \\
(0 \%)\end{array}$ \\
\hline
\end{tabular}

producciones de /b/, /d/ y /g/ se realizan de manera aproximante, y en los casos de la fricativa alveolar en posición de coda, de manera sonora. Este tipo de realizaciones también son las que predominan entre verbo y objeto. Se observa alternancia en las realizaciones de nombre + adjetivo, donde se da una producción espirantizada

Figura 6. Producción sonora o sonorizada de /s/ (\%) en los distintos contextos sintácticos. $\mathrm{M}=$ mujer; $\mathrm{H}=$ hombre.

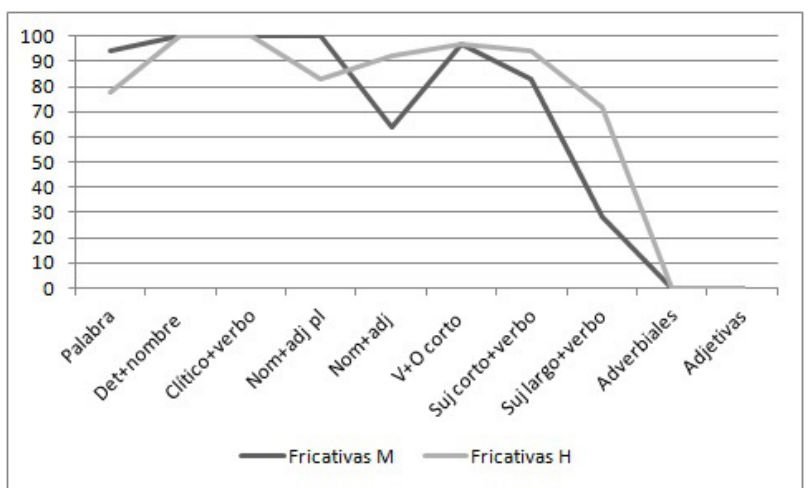


en casi el $100 \%$ de casos, lo que indicaría que no existe frontera. Sin embargo, en el caso de la sonorización de /s/, parece que hay diferencia entre los dos informantes: en la mujer se observa mayor variación, por lo que en su caso sí podría existir una frontera. En el contexto de sujeto corto + verbo se aprecia un descenso en la espirantización en el caso de la mujer frente al del hombre, en el que no disminuye el porcentaje de espirantización hasta los contextos de sujeto largo + verbo. En ambos informantes, se bloquean los procesos de forma considerable entre el sujeto largo y el verbo y entre sujeto corto y verbo en el caso de la mujer también. En los casos de cláusulas parentéticas (adverbiales y adjetivas), se observa un $100 \%$ de realizaciones oclusivas para /b/, /d/ y /g/, y de sordas para la fricativa alveolar en posición de coda. La variación observada, tanto con respecto a la diferencia entre sujetos como en relación con ciertos contextos (por ejemplo, nombre + adjetivo) no se puede explicar dado el tamaño reducido de la muestra. Para poder establecer conclusiones es necesario ampliar la muestra.

\section{DISCUSIÓN}

Tal como afirma Parrell (2011), tanto en interior de palabra como en frontera de palabra, la menor duración de la bilabial /b/ es el resultado de una menor constricción de los órganos articuladores y, por tanto, se produce espirantización, mientras que la mayor duración del gesto en la frontera de un grupo prosódico mayor da como resultado un cierre completo porque los articuladores tienen tiempo para cerrarse y, por tanto, se realiza la oclusión. La investigación presente no considera el origen de la espirantización (o de la sonorización de /s/), pero acepta la sugerencia de Parrell de que las fronteras prosódicas mayores bloquean la realización del proceso y, efectivamente, esto es lo que se ha descubierto tras analizar los resultados del presente experimento.

Así pues, los datos del análisis sobre los procesos fonológicos segmentales parecen indicar que estos se pueden utilizar como parámetros válidos para postular la existencia de fronteras prosódicas de marca de sintagma fonológico en español. Tras haber analizado la realización oclusiva o aproximante de las obstruyentes sonoras $(/ \mathrm{b} /, / \mathrm{d} /, / \mathrm{g} /)$ y la realización sorda o sonora de la fricativa alveolar (/s/) en posición de coda en español, se ha podido constatar un comportamiento fonético diferente en ciertas estructuras sintácticas en las que dichos segmentos se producen (menor cohesión sintáctica frente a mayor cohesión sintáctica), lo cual parece demostrar la existencia de una frontera prosódica que está bloqueando la realización de cualquiera de los dos procesos. Estos resultados pueden servir para establecer los límites de los sintagmas fonológicos en español, cuya existencia no había sido probada hasta ahora en esta lengua mediante pruebas segmentales.

Como ya se ha indicado anteriormente, $/ \mathrm{b} /, / \mathrm{d} / \mathrm{y} / \mathrm{g} / \mathrm{se}$ comportan como una clase homogénea, al menos en cuanto a lo analizado en estas páginas: tanto los análisis estadísticos como el porcentaje de espirantización son similares para los tres sonidos. No obstante, llama la atención que, en los casos de la bilabial /b/ en contacto con el determinante masculino, la producción de oclusivas es superior a lo esperado, por lo que los gráficos muestran un ligero descenso en determinante + nombre. No se han podido establecer conclusiones a este respecto, pues la bibliografía consultada no alude en ningún momento a una realización oclusiva de la bilabial tras /1/, de manera similar a lo que ocurre en español con /d/ tras /1/. Es interesante resaltar que Campos-Astorkiza (2015) sugiere que la sonorización /s/ se reduce en presencia de sonantes. Si esto ocurre así en el caso de /s/, el hecho de que disminuya la espirantización de la bilabial /b/ tras /1/, al igual que ocurre en la dental /d/ tras $/ 1 /$, también podría explicarse por el carácter sonante de la /1/. Es decir, esto implicaría que el aumento de oclusión en las obstruyentes o la reducción de sonorización en la fricativa deberían revisarse en relación con las categorías obstruyente/sonante, pues estas pueden tener una influencia mayor de lo que hasta ahora se pensaba.

El análisis de las distintas estructuras sintácticas que se ha llevado a cabo para los segmentos obstruyentes sonoros /b/, /d/ y /g/ y para la fricativa alveolar sorda en posición de coda /s/ ha mostrado que la realización aproximante de las primeras y la sonorizada de la segunda va disminuyendo a medida que la estructura sintáctica se hace más compleja. La menor presencia, o directamente la ausencia, de realización aproximante de las obstruyentes y de sonorización de la alveolar en estos contextos (por debajo del $70 \%$ ) permite hipotetizar que existe una frontera prosódica, que bloquea el proceso, lo cual implica que la palabra que contiene $/ \mathrm{b}, \mathrm{d}, \mathrm{g} / \mathrm{y} / \mathrm{s} /$, y la palabra precedente o siguiente respectivamente, se encuentran en constituyentes o dominios prosódicos distintos.

Los resultados de esta investigación indican que la unión de determinante + nombre y clítico + verbo es estable y muy fuerte, ya que prácticamente en todos los contextos sintácticos más cohesionados se observa una realización aproximante de las obstruyentes sonoras y una realización sonora o sonorizada de la fricativa alveolar sorda. No obstante, esta unión tan fuerte puede ser indicativa de una unión en un límite prosódico inferior, el de la palabra prosódica, ya que la ausencia de una frontera prosódica en sí misma no prueba una unión prosódica en el límite del sintagma fonológico. Con los datos aportados en este trabajo no se puede resolver esta cuestión y, por tanto, sigue sin resolverse, por ejemplo, la doble adjunción propuesta por Elordieta (2014) para los clíticos y los determinantes, posible tanto en el nivel de la palabra prosódica como en el del sintagma fonológico.

Por otra parte, llama la atención la variación observada en los casos de nombre + adjetivo. La mayor diferencia entre las obstruyentes sonoras y la fricativa alveolar radica en que, en las primeras, se observa mayor unión entre las secuencias de nombre y adjetivo, pues se constata un porcentaje mayor de espirantización, mientras que, en el caso de la fricativa, se ha comprobado una unión mayor en el caso del informante hombre, pero no así en el 
de la mujer. En las secuencias de nombre + adjetivo, cuando la /s/ es marca de plural, se observa un porcentaje muy elevado de sonorización.

La teoría prosódica de Nespor y Vogel (1986/1994, 2007) propone que entre un núcleo y el material especificador situado en su lado no recursivo no existe una frontera de sintagma fonológico; en el caso del español, este podría ser el caso de los adjetivos prenominales, como por ejemplo en verde perro, lo cual explicaría los resultados obtenidos.

Sin embargo, esta secuencia formaría dos sintagmas fonológicos diferentes si el núcleo se encontrase a la izquierda y los complementos a la derecha, como en perro verde. La variación observada en los datos analizados - menor presencia de sonorización cuando la /s/ es parte de la raíz y mayor presencia de sonorización cuando es el morfema plural- se puede deber al pequeño tamaño de la muestra, pero también puede ser consecuencia de que esta representación no sea siempre así y, a pesar de que el núcleo esté a la izquierda, en algunos casos los hablantes lo representen como un único sintagma fonológico. Para dar respuesta a los interrogantes suscitados por estos datos habría que contrastar sintagmas del tipo niña guapa y pequeño gato. En todo caso, esta cuestión se tendrá en cuenta para futuros trabajos, pues incluso en la teoría sintáctica no existe acuerdo acerca de la relación entre nombre y adjetivo.

En los casos en los que /s/ es un morfema, se ha observado una unión muy fuerte entre el nombre y el adjetivo en plural, lo cual puede relacionarse con la propia concordancia sintáctica, que provoca que se representen dentro del mismo grupo prosódico, al igual que determinante y nombre, y clítico y verbo cuando son plurales. La existencia de dos marcas morfológicas o sintácticas dentro del mismo grupo prosódico es prueba suficiente para que su unión se realice en el nivel del sintagma fonológico, no en el de la palabra prosódica, según la hipótesis de Lleó (2006). Los resultados obtenidos parecen corroborar esta hipótesis.

Se ha observado una unión fuerte en los casos de verbo + objeto, sobre todo cuando el objeto es corto (una única palabra), lo que parece indicar, entonces, que verbo y objeto forman un único sintagma fonológico. La explicación que ofrecen Ladd (1986) y Selkirk (1986) para ello se basa en el algoritmo Align-XP, R, es decir, el alineamiento del lado derecho de un sintagma sintáctico con el lado derecho de un sintagma fonológico, lo que en español supone que la frontera de sintagma fonológico se crea a partir de los límites derechos de los sintagmas, de modo que no puede existir frontera entre verbo y objeto, tal como se manifiesta en los resultados obtenidos.

Lo mismo ocurre en las estructuras con un sintagma preposicional ( $\mathrm{SN}+\mathrm{SPrep}$ ), que no ponen de manifiesto que exista una frontera prosódica entre el sintagma nominal precedente y el sintagma preposicional. Para Nespor y Vogel $(1986 / 1994,2007)$ estas estructuras, del tipo SPrep, sí forman un sintagma fonológico independiente del sintagma nominal precedente, pues poseen más de una palabra prosódica y un acento primario, de modo que en los datos estudiados debería haberse constatado una frontera prosódica. No obstante, la explicación de que no haya sido así parece encontrarse en que se produce un alineamiento solo en el lado derecho de los constituyentes sintácticos y el sintagma fonológico (Selkirk, 1986; Truckenbrodt, 1995, 1999). Son necesarios análisis más profundos sobre estas secuencias para dar respuesta a todos estos interrogantes.

Se ha podido confirmar, en cambio, la existencia de una frontera estable entre sujeto y verbo, sobre todo en los casos más largos de sujeto, ya que tanto para las obstruyentes sonoras como para la fricativa alveolar, se observa un bloqueo de la espirantización o de la sonorización respectivamente. En los casos de sujeto corto + verbo también ocurre esto en la mujer para las obstruyentes sonoras. En este sentido, se puede afirmar que la estructura sujeto + verbo no forma un sintagma fonológico único, debido al bloqueo del proceso, consecuencia de una frontera, de manera que sujeto y verbo pertenecen a dos unidades prosódicamente diferenciadas, sobre todo cuando el sujeto es largo. Para afirmar esto con rotundidad en los casos de sujeto corto se necesitan más estudios.

Además, se ha observado un límite prosódico claro en las cláusulas de menor cohesión sintáctica, adverbiales y adjetivas, que se corresponde con un límite de sintagma entonativo, dado que en todos los casos analizados se ha producido una oclusiva o una fricativa alveolar sorda, sin haberse documentado ningún caso de espirantización o de sonorización. No obstante, la presencia de un límite prosódico aquí se debe a la existencia de un constituyente prosódico inmediatamente superior, como puede ser el sintagma entonativo, ya que se corresponde con contornos entonativos completos al presentar un tono de frontera, como han descrito otros autores (Prieto, 2006).

Por todo ello, el análisis llevado a cabo en estas páginas parece demostrar que los procesos fonológicos recurrentes en español constituyen pruebas válidas para comprobar la existencia de constituyentes prosódicos en esta lengua. Queda para trabajos futuros corroborar y afianzar los datos aquí obtenidos. Además, se podrían tener en cuenta otros procesos, como la aspiración de la fricativa alveolar /s/ en posición de coda, puntos de articulación de las nasales y el resilabeo, con el objetivo de descubrir los límites prosódicos en los que estos fenómenos se producen; porque si la variación prosódica no se puede predecir meramente a partir de una teoría de la alofonía en las obstruyentes oclusivas sonoras (tal como afirma Parrell, 2011, p. 442), tampoco ocurrirá así, cabe suponer, con respecto al resto de procesos fonológicos.

\section{CONCLUSIONES}

Tras el análisis de dos procesos fonológicos existentes en español (la espirantización de las obstruyentes sonoras y la sonorización de la fricativa alveolar sorda en posición de coda) en los distintos constituyentes sintácticos, se ha descubierto que /b/, /d/ y /g/ se comportan como una misma clase. Utilizar ambos procesos segmentales para 
delimitar los sintagmas fonológicos en español solo ha permitido establecer con certeza una marca prosódica de sintagma fonológico entre sujeto (largo) y verbo. La ausencia de una frontera prosódica en estructuras sintácticas inferiores se debe a los límites de palabras prosódicas. La existencia de las fronteras prosódicas descubiertas en estructuras superiores se debe a la frontera de los sintagmas entonativos. No obstante, para poder ampliar las conclusiones y corroborar la propuesta de este trabajo, se necesita ampliar la muestra a un número mayor de informantes.

\section{AGRADECIMIENTOS}

Me gustaría agradecer a los informantes su participación, pues se prestaron voluntariamente para realizar las grabaciones; sin ellos no habría podido realizar este trabajo. También quiero agradecer a Rebeka Campos-Astorkiza que me facilitara la bibliografía, y a los revisores anónimos sus útiles comentarios, que sin duda han mejorado el trabajo. En especial quiero mostrar mi agradecimiento a Gorka Elordieta por atender mis numerosas consultas y por sus acertadas sugerencias.

\section{REFERENCIAS}

Battaner, E., Carbó, C., Gil, J., Llisterri, J., Machuca, M. J., Madrigal, N., Marrero, V., de la Mota, C., Riera, M., \& Ríos, A. (2007). VILE: Estudio acústico de la variación inter e intralocutor en español. En M. González González, E. Fernández Rei y B. González Rei (Eds.), Actas del III Congreso Internacional de Fonética Experimental (pp. 157-167). Santiago de Compostela: Xunta de Galicia.

Beckman, M. E., \& Pierrehumbert, J. B. (1986). Intonational structure in Japanese and English. Phonology Yearbook, 3, 255-309.

Benet Parente, A. (2012). El fraseig prosòdic en la parla espontània del català $i$ del castellà (tesis doctoral). Universität Hamburg.

Booij, G. (1988). [Reseña del libro Prosodic phonology, de Marina Nespor y Irene Vogel]. Journal of Linguistics, 24, 515-525.

Campos-Astorkiza, R. (2010). Voicing assimilation and prosodic structure in Spanish. The Journal of the Acoustical Society of America, 128, 2288. http://dx.doi.org/10.1121/1.3508028

Campos-Astorkiza, R. (2014). Sibilant voicing assimilation in Peninsular Spanish as gestural blending. En M. H. Côté y E. Mathieu (Eds.), Variation within and across Romance languages: Selected papers from the 41st Linguistic Symposium on Romance Languages (LSRL), Ottawa, 5-7 May 2011 (17-38). Amsterdam: John Benjamins. http://dx.doi.org/10.1075/ cilt. $333.04 \mathrm{cam}$

Campos-Astorkiza, R. (2015). Segmental and prosodic conditionings on gradient voicing assimilation in Spanish. En R. Klassen, J. M. Liceras y E. Valenzuela (Eds.), Hispanic linguistics at the crossroads: Theoretical linguistics, language acquisition and language contact. Proceedings of the Hispanic Linguistics Symposium 2013 (pp. 127-144). Amsterdam: John Benjamins. http://dx.doi.org/10.1075/ihll.4.07cam

Dresher, B. E. (1996). Introduction to metrical and prosodic phonology. En J. L. Morgan y K. Demuth (Eds.), Signal to syntax: Bootstrapping from speech to grammar in early acquisition (pp. 41-54). Mahwah, NJ: Lawrence Erlbaum.

Elordieta, G. (2014). The word in phonology. En I. Ibarretxe-Antuñano y J. L. Mendívil-Giró (Eds.), To be or not to be a Word:
New reflections on the definition of word (pp. 6-65). Newcastle upon Tyne: Cambridge Scholars Publishing.

Gili Gaya, S. (1950/1975). Elementos de fonética general (5. $\left.{ }^{\mathrm{a}} \mathrm{ed}\right)$. Madrid: Gredos.

Goldsmith, J. A. (1981/2000). Las unidades subsegmentales en la fonología española: Enfoque autosegmental. En J. Gil Fernández (Ed., Trad.), Panorama de la fonología española actual (pp. 337-356). Madrid: Arco/Libros. (Traducción de Subsegmentals in Spanish phonology: An autosegmental approach. En W. W. Cressey y D. J. Napoli, Eds., Linguistic Symposium on Romance Languages 9, pp. 1-16. Washington, D.C.: Georgetown University Press)

González, C. (2006). The phonetics and phonology of spirantization in North-central Peninsular Spanish. Anuario del Seminario de Filología Vasca «Julio de Urquijo»[ASJU], 40, 409-436. Disponible en http://www.ehu.eus/ojs/index.php/ASJU/article/view/4398

Harris, J. W. (1984/2000). La espirantización en castellano y la representación fonológica autosegmental. En J. Gil Fernández (Ed.), Panorama de la fonología española actual (pp. 357371). Madrid: Arco/Libros. (Reimpresión de Estudis Gramaticals I. Working Papers in Linguistics, pp. 149-167. Universitat Autònoma de Barcelona)

Hayes, B. (1989). The prosodic hierarchy in meter. En P. Kiparsky y G. Youmans (Eds.), Phonetics and phonology, Vol. 1: Rhythm and meter (pp. 201-260). San Diego, CA: Academic Press. http://dx.doi.org/10.1016/B978-0-12-409340-9.50013-9

Hayes, B. (1995). Metrical stress theory: Principles and case studies. Chicago, IL: The University of Chicago Press.

Hualde, J. I. (2003). El modelo métrico y autosegmental. En P. Prieto (Coord.), Teorías de la entonación (pp. 155-184). Barcelona: Ariel.

Hualde, J. I., Shosted, R., \& Scarpace, D. (2011). Acoustics and articulation of Spanish /d/ spirantization. En Proceedings of the 17th International Congress of Phonetic Sciences (ICPhS 17Hong Kong, China), 906-909.

Hualde, J. I., Simonet, M., \& Nadeu, M. (2011). Consonant lenition and phonological recategorization. Laboratory Phonology, 2(2), 301-329. http://dx.doi.org/10.1515/LABPHON.2011.011

Ito, J., \& Mester, A. (2009). The extended prosodic word. En J. Grijzenhout y B. Kabak (Eds.), Phonological domains: Universals and deviations (pp. 135-194). Berlin: Mouton de Gruyter.

Ito, J., \& Mester, A. (2013). Prosodic subcategories in Japanese. http://dx.doi.org/10.1016/j.lingua.2012.08.016

Ladd, D. R. (1986). Intonational phrasing: The case for recursive prosodic structure. Phonology Yearbook, 3, 311-340.

Landis, J. R., \& Koch, G. G. (1977). The measurement of observer agreement for categorical data. Biometrics, 33(1), 159-174.

Lleó, C. (2006). Early acquisition of nominal plural in Spanish. Catalan Journal of Linguistics, 5, 191-219.

Martínez Celdrán, E. (1984). Fonética. Barcelona: Teide.

Martínez Celdrán, E. (2013). Caracterización acústica de las aproximantes espirantes en español. Estudios de Fonética Experimental, 22, 11-35.

Martínez Celdrán, E., \& Fernández Planas, A. M. (2007). Manual de fonética española. Barcelona: Ariel.

McCarthy, J. J., \& Prince, A. S. (1986). Prosodic morphology. Amherst, MA: University of Massachusetts.

Nespor, M., \& Vogel, I. (1986/1994). La prosodia (A. Ardid Gumiel, Trad.). Madrid: Visor. (Trabajo original: Prosodic phonology. Dordrecht: Foris)

Nespor, M., \& Vogel, I. (2007). Prosodic phonology. With a new foreword. Berlin: Mouton de Gruyter.

Nibert, H. J. (2000). Phonetic and phonological evidence for intermediate phrasing in Spanish intonation (tesis doctoral). University of Illinois at Urbana-Champaign. http://hdl.handle. net/2142/77481

Ortega-Llebaria, M. (2004). Interplay between phonetic and inventory constraints in the degree of spirantization of voiced stops: Comparing intervocalic /b/ and intervocalic /g/ in Spanish and English. En T. L. Face (Ed.), Laboratory approaches to Spanish phonology (pp. 237-254). Berlin: Mouton de Gruyter. 
Pak, M. (2008). The postsyntactic derivation and its phonological reflexes (tesis doctoral). Disponible en http://repository.upenn. edu/dissertations/AAI3328631

Parrell, B. (2011). Dynamical account of how /b, d, g/ differ from /p, t, k/ in Spanish: Evidence from labials. Laboratory Phonology, 2(2), 423-449. http://dx.doi.org/10.1515/labphon.2011.016

Pater, J., \& Paradis, J. (1996). Truncation without templates in child phonology. En A. Stringfellow, D. Cahana-Amitay, E. Hughes y A. Zukowski (Eds.), Proceedings of the 20th annual Boston University Conference on Language Development (BUCLD 20), Vol. 2 (pp. 540-551). Somerville, MA: Cascadilla Press.

Peperkamp, S. (1997). Prosodic words. HIL Dissertations 34. The Hague: Holland Academic Graphics.

Prieto, P. (2006). Phonological phrasing in Spanish. En F. MartínezGil y S. Colina (Eds.), Optimality-theoretic advances in Spanish phonology (pp. 39-61). Amsterdam: John Benjamins. http:// dx.doi.org/ 10.1075/la.99.03pri

Quilis, A. (1981). Fonética acústica de la lengua española. Madrid: Gredos.

Quilis, A. (1993). Tratado de fonología y fonética españolas. Madrid: Gredos.

Real Academia Española (2011). Nueva gramática de la lengua española. Fonética y fonología. Madrid: Espasa-Calpe.

Samuels, B. D. (2011). Phonological architecture: A biolinguistic perspective. Oxford, UK: Oxford University Press.

Scheer, T. (2011). A guide to morphosyntax-phonology interface theories. Berlin: De Gruyter Mouton.

Schmidt, L. B., \& Willis, E. W. (2011). Systematic investigation of voicing assimilation of Spanish /s/ in Mexico City. En S. M. Alvord (Ed.), Selected Proceedings of the 5th Conference on Laboratory Approaches to Romance Phonology (pp. 1-20). Somerville, MA: Cascadilla Proceedings Project.

Selkirk, E. O. (1978/1981). On prosodic structure and its relation to syntactic structure. En T. Fretheim (Ed.), Nordic prosody
II: Papers from a symposium (pp. 111-140). Trondheim: Tapir.

Selkirk, E. O. (1984). Phonology and syntax: The relation between sound and structure. Cambridge, MA: MIT Press.

Selkirk, E. O. (1986). On derived domains in sentence phonology. Phonology Yearbook, 3, 371-405. http://dx.doi.org/10.1017/ S0952675700000695

Selkirk, E. O. (2001). Syntax-phonology interface. En International encyclopedia of the social and behavioral sciences [Linguistics section edited by B. Comrie], pp. 15407-15412. Amsterdam: Elsevier.

Selkirk, E. O. (2011). The syntax-phonology interface. En J. Goldsmith, J. Riggle y A. C. L. Yu (Eds.), The handbook of phonological theory, second edition (pp. 435-484). Oxford, UK: WileyBlackwell. http://dx.doi.org/10.1002/9781444343069.ch14

Selkirk, E. O., \& Tateishi, K. (1988). Constraints on minor phrase formation in Japanese. En L. MacLeod, G. Larson y D. Brentari (Eds.), Proceedings of the 24th Annual Meeting of the Chicago Linguistic Society (pp. 316-336). Chicago, IL: Chicago Linguistic Society.

Selkirk, E. O., \& Tateishi, K. (1991). Syntax and downstep in Japanese. En C. Georgopoulos y R. Ishihara (Eds.), Interdisciplinary approaches to language. Essays in honor of S.-Y. Kuroda (pp. 519-543). Dordrecht: Kluwer. http://dx.doi.org/10.1007/ 978-94-011-3818-5 28

Truckenbrodt, H. (1995). Phonological phrases: Their relation to syntax, focus, and prominence (tesis doctoral). Massachusetts Institute of Technology. Disponible en http://www.ai.mit.edu/ projects/dm/theses/truckenbrodt95.pdf

Truckenbrodt, H. (1999). On the relation between syntactic phrases and phonological phrases. Linguistic Inquiry, 30(2), 219-255.

Vigário, M. (2010). Prosodic structure between the prosodic word and the phonological phrase: Recursive nodes or an independent domain? The Linguistic Review, 27(4), 485-530. http:// dx.doi.org/10.1515/tlir.2010.017 